\title{
Educación inclusiva en el nivel medio-superior: análisis desde la perspectiva de directores
}

\section{Inclusive education in Mexican high schools: an analysis from the principals' standpoint}

\author{
IVANIA DE LA CRUZ OROZCO*
}

En este artículo se analizan las estrategias que diseñan e implementan directores de bachilleratos públicos en México para promover servicios educativos inclusivos a jóvenes con alguna discapacidad. Utilizando como marco analítico la Guía para asegurar la inclusión y la equidad en la educación, elaborada por la Unesco y adoptada por las autoridades educativas mexicanas para el periodo 2012-2018, se examinan 76 testimonios de directores recabados a través de una encuesta en línea. Los hallazgos muestran que estos recurren a una gama diversa de estrategias para fomentar una educación inclusiva; sin embargo, también es evidente que no disponen de los recursos necesarios para hacerlo cabalmente, pues las dependencias públicas a cargo de la política educativa no han transitado del reconocimiento del derecho a una educación inclusiva a la puesta en marcha de sistemas de financiamiento y acompañamiento que garanticen ese derecho en la práctica.

This article explores the strategies implemented by public high school principals to provide education to students who tend to be excluded from this service: Students with disabilities. Data comes from an online survey conducted in 2017 to gather information about the activities of public high school principals in Mexico. We analyzed the testimonies of 76 high school principals using as the Guide for ensuring inclusion and equity in education, designed by Unesco's and adopted by Mexican educational authorities to promote an inclusive education for all. The findings show that high school principals implement a range of proactive strategies to promote an inclusive education. However, testimonies also show principals are not fully supported by educational authorities, which have not moved from discourse to action. This means schools lack the resources to provide a high-quality education to students with disabilities.
Palabras clave: educación inclusiva, liderazgo escolar, estudiantes con discapacidad, estudiantes en desventaja, educación media superior

\section{Key words:}

inclusive education, school leadership, students with disabilities, disadvantaged students, high school

Recibido: 18 de febrero de 2019| Aceptado para su publicación: 10 de febrero de 2020

Publicado: 28 de febrero de 2020

Recuperado de: https://sinectica.iteso.mx/index.php/SINECTICA/article/view/978 doi: $10.31391 /$ S2007-7033(2020)0054-008

\footnotetext{
* Doctora en Desarrollo Educativo (International Educational Development, Columbia University). Investigadora Cátedra Conacyt-CIDE. Programa Interdisciplinario de Política y Prácticas Educativas. Líneas de investigación: escuelas en comunidades, educación de la población indígena en México y expansión de la educación media superior en México. Correo electrónico: ivania.delacruz@cide.edu
} 


\section{INTRODUCCIÓN}

7 concepto de educación inclusiva está incorporado al discurso de las au- toridades educativas mexicanas, pero poco sabemos acerca de los avances y desafíos de su implementación desde la perspectiva de quienes lideran las prácticas cotidianas y los procesos de cambio en las escuelas: los directores o las directoras. Existe literatura que discute definiciones de educación inclusiva y su evolución para entender la diversidad de formas que toma, así como para destacar el rol que juega la escuela en su vivencia y promoción. Los estudios recientes sobre inclusión hacen hincapié en lo que la escuela debe promover para garantizar atención y participación de todos los estudiantes, sin importar sus características, y dejan de centrar la discusión en aquello que los estudiantes no tienen o no hacen.

También hay estudios que proponen metodologías para diagnosticar la situación de la educación en entornos diversos, que van desde los sistemas educativos nacionales hasta las escuelas, en particular de nivel básico y superior (Flores y García, 2016; Varas, 2018). Sin embargo, hay pocos análisis respecto a la situación, avances y desafíos de la educación inclusiva en el nivel medio superior, el cual, en 2012, se integró a la educación obligatoria brindada por el Estado mexicano.

Si bien se sabe del grado de inclusividad en este nivel educativo en términos de disponibilidad de escuelas (cobertura), no existen estudios sobre la educación inclusiva como parte de las dinámicas cotidianas o de una inclusión entendida como un conjunto de principios transversales que guíen la práctica escolar. Lo que sabemos se reduce a la existencia de términos en la normativa nacional o a la existencia de programas nacionales que buscan promover una educación inclusiva, sin que en realidad se conozca la efectividad de esos programas. En este sentido, son necesarios estudios que describan en qué medida la educación inclusiva deja de existir como algo impuesto o discursivo, y se encuentra en la agenda de actividades cotidianas de quienes integran las comunidades escolares.

Este artículo constituye una contribución a esta laguna en la investigación educativa. A partir de datos provenientes de una encuesta a directores/as de educación media superior (EMS) de escuelas públicas en México, analizamos dos aspectos de la inclusión educativa en sus establecimientos escolares: los estudiantes que, según los directores/as escolares, no se benefician de la educación inclusiva (en este caso, los estudiantes con alguna discapacidad) y las estrategias usadas por las figuras directivas para brindar a estos estudiantes el acceso a una educación inclusiva. El primer tema hace referencia a cómo los directores/ as definen y describen los eventos y situaciones que ameritan intervenciones para promover una educación inclusiva. El segundo refiere cómo el personal directivo de EMS implementa u opera estrategias de inclusión.

El artículo se estructura en cinco secciones. Primero, hacemos una revisión de literatura sobre la educación inclusiva, así como un diagnóstico de la presencia de ese concepto en las leyes y los programas que guiaron la política educativa en México de 2012 a 2018. Este apartado también incluye la descripción del marco analítico usado para entender cómo los directores/as escolares definen y abordan el tema de la educación inclusiva. La segunda sección contiene la estrategia metodológica para recabar y analizar los datos usados en esta investigación.

De la Cruz. Educación inclusiva en el nivel medio-superior: análisis desde la perspectiva de directores Sinéctica 54 www.sinectica.iteso.mx 
Posteriormente, compartimos los resultados sobre cómo los directores/as de EMS buscan brindar una educación inclusiva a estudiantes con alguna discapacidad, un apartado de conclusiones y las referencias consultadas.

\section{MARCo TEÓRICO Y REVISIÓN DE LITERATURA}

\section{Definiciones de la educación inclusiva}

El concepto de educación inclusiva tuvo un auge en su desarrollo a partir de estudios y programas educativos que examinaron la situación que enfrentaba la población infantil con discapacidad o necesidades especiales. Estos análisis pusieron en evidencia la falta de estrategias para garantizar el derecho a una educación de calidad entre estas poblaciones y provocaron una discusión más amplia del concepto en tres sentidos. Primero, promovieron la idea de que crear espacios aislados para atender exclusivamente a poblaciones con necesidades especiales generaba una consecuencia negativa: la segmentación de sistemas educativos y el aislamiento de las poblaciones atendidas. Segundo, el concepto de educación inclusiva se vio como un objetivo no solo para los estudiantes con capacidades especiales, sino para la diversidad de estudiantes que integran las comunidades escolares, en particular para quienes han enfrentado barreras de acceso a la educación en forma sistemática: minorías étnicas y lingüísticas, poblaciones con desventajas académicas, migrantes, mujeres, entre otros. Tercero, fue evidente que era necesario deconstruir los supuestos sobre las condiciones que favorecen la educación inclusiva (Moliner, 2013). En este sentido, la discusión dejó de suponer que son los estudiantes quienes deben adaptarse a las características de la escuela para dar paso al principio de que son las escuelas las que deben ajustarse para atender a todos los estudiantes, sin importar su origen y características.

\section{Educación inclusiva en México}

Las características actuales de la educación inclusiva representan un desafío importante para las autoridades educativas. Cuando el parámetro para diagnosticar esta educación era solo el nivel de cobertura, bastaba con la presencia de un recinto escolar para mostrar avances en este rubro de la política educativa. Esto no quiere decir que dicha meta se haya cumplido en todos los sistemas educativos nacionales o en todos los niveles de educación obligatoria. De hecho, en México existen poblaciones en condición de desventaja (la población indígena, la que presenta discapacidad, la que reside en localidades rurales, la que se encuentra en condición de pobreza) que tienen mayores probabilidades de no asistir a la escuela (INEE, 2019).

En el nivel escolar que analizamos, la EMS aún está lejos de los niveles de cobertura total que se plantearon para el ciclo escolar 2022, hay poca información confiable sobre el acceso a la educación para población con necesidades especiales y se sabe que en el 55\% de los planteles no hay rampas de acceso para población con discapacidad motriz (INEE, 2018). 
La ampliación de la población que tiene el derecho a recibir educación inclusiva y el enfoque en la transformación escolar para garantizar ese derecho hacen necesaria una política educativa nacional que no solo establezca la importancia de la educación inclusiva, sino que impulse su consecución con programas de política pública bien diseñados, financiados y que cuenten con el involucramiento de funcionarios locales, así como con quienes integran las comunidades escolares.

Para el periodo analizado (2012-2018), en México, la Constitución determina en su artículo $3^{\circ}$ que toda persona tiene derecho a recibir educación de calidad. En concordancia, la Ley General de Educación prevé que la educación impartida por el Estado mexicano debe "promover inclusión”, "fomentar la valoración de la diversidad y la cultura de inclusión" y "permitir al educando su plena inclusión y participación en la sociedad". Además, esta ley obliga a las autoridades educativas a "fortalecer la educación especial y la educación inicial, incluyendo a las personas con discapacidad".

Respecto a la educación especial, la Ley General de Educación establece que su propósito es "identificar, prevenir y eliminar las barreras que limitan el aprendizaje y la participación plena y efectiva en la sociedad de las personas con discapacidad, con dificultades severas de aprendizaje, de conducta o de comunicación, así como de aquellas con aptitudes sobresalientes". Esta educación deberá garantizarse en "un contexto educativo incluyente, que se debe basar en los principios de respeto, equidad, no discriminación, igualdad sustantiva y perspectiva de género" y tendrá lugar en escuelas regulares, o bien, en establecimientos de educación especial (Ley General de Educación, 2018).

La implementación de estos requerimientos legales en el sexenio 2012-2018 se impulsó a través del modelo educativo para la educación obligatoria o nuevo modelo educativo, el cual contaba en el diseño con un apartado sobre la educación inclusiva llamado "Inclusión y equidad". Este componente establecía que los planteamientos curriculares, prácticas, métodos educativos, materiales y ambientes escolares debían obedecer a la lógica de inclusión y equidad (SEP, 2017, p. 152).

Para lograr este objetivo, se propuso, en primer lugar, un planteamiento curricular incluyente, es decir, contar con un currículo flexible que fomentara el aprecio por la diversidad, eliminara la discriminación y entregara con oportunidad los libros de texto. En segundo lugar, se destacó la necesidad de garantizar condiciones equitativas para las escuelas con mejoras en su infraestructura y equipamiento, en sus prácticas de gestión y sistemas de participación, y en el sistema de supervisión y apoyo por parte de autoridades educativas. En tercer lugar, se planteó garantizar educación pertinente y de calidad a estudiantes indígenas y a hijos de jornaleros migrantes, y en cuarto, completar la transición de educación especial a educación inclusiva, lo cual se entiende como asegurar una oferta educativa pertinente para estudiantes superdotados; eliminar las barreras de acceso que los estudiantes con discapacidad enfrentan para asistir a una escuela regular; formar a docentes para que trabajen con base en los principios de la educación inclusiva; mejorar la infraestructura y tecnologías disponibles en las escuelas; implementar estrategias para alcanzar la igualdad de género; y seguir otorgando becas que permitan a todos los estudiantes completar sus trayectorias escolares (SEP, 2017). 


\section{La inclusión educativa en la EMS}

Respecto a la EMS, existen dos documentos sobre política educativa que consideran la educación inclusiva: Directrices para mejorar la permanencia en EMS y Condiciones básicas para la enseñanza y el aprendizaje en los planteles de educación media superior en México, ambos elaborados por el Instituto Nacional para la Evaluación Educativa (INEE). En ellos, los conceptos relacionados con educación inclusiva se refieren a la formación docente para una educación inclusiva e intercultural; las desventajas académicas de la población indígena; y la desventaja en infraestructura en subsistemas que atienden a poblaciones en zonas rurales (INEE, 2017, 2018).

Considerando la normativa, programas de política pública y estudios sobre evaluación educativa, en este artículo analizamos una población considerada en riesgo de no ver garantizado su derecho a una educación inclusiva: los estudiantes con alguna discapacidad. Para el grupo en edad de cursar EMS, los porcentajes más altos de inasistencia escolar se presentan entre quienes tienen alguna discapacidad, junto con quienes viven en los hogares con menor ingreso o en zonas rurales (UNICEF, 2016). Los servicios públicos educativos del nivel medio superior se imparten a través de los centros de atención para estudiantes con discapacidad (CAED). El trabajo en estos se basa en un modelo que, enmarcado en los derechos humanos, busca promover prácticas educativas que combatan la discriminación y la exclusión, reconozcan en "el otro" el derecho a la diferencia y garanticen el derecho a la educación de calidad entre la población con discapacidad (SEP, 2018).

De acuerdo con la literatura que sugiere no segregar los sistemas educativos y crear distintos sistemas para diferentes tipos de estudiantes, los CAED debían instalarse dentro de bachilleratos que llevaran tiempo funcionando, aunque constituían un modelo de educación no escolarizada. A pesar de la importancia de garantizar educación de calidad a estudiantes con discapacidad, hoy se desconoce en qué medida el programa de los CAED tiene un diseño adecuado o si se está implementando de tal manera que en las comunidades escolares se haya generado una cultura de la inclusión. Esta investigación es un primer acercamiento a estos temas desde la perspectiva de los directores/as de EMS.

La literatura sobre educación a personas con discapacidad nos permite destacar los beneficios que todos los estudiantes obtienen con prácticas de educación inclusiva (Iacoboni y Moirano, 2018). Además, hay estudios que muestran apertura de parte de estudiantes y docentes hacia la inclusión (De la Cruz, 2018). Sin embargo, la literatura también resalta algunas dificultades y retos que los docentes y estudiantes sin discapacidad enfrentan cuando se integran estudiantes con capacidades diferentes a los espacios escolares. Estas dificultades y resistencias a la inclusión están ligadas a la falta de tiempo, recursos, apoyo, coordinación entre docentes, colaboración con padres y rigidez institucional (García y Villar, 1987; Illán, 1989; Dengra, Durán y Verdugo, 1991, citados en Arrebola y Villuendas, 2004; Sanhueza, Granada y Bravo, 2012).

Respecto a las dificultades que enfrentan los docentes, algunos estudios reportan que estos actores experimentan sensaciones de estrés, ansiedad o frustración ante el mandato de integrar a estudiantes con discapacidad a los cursos que toman los estudiantes sin discapacidades (Iacoboni y Moirano, 2018; Varas, 
2018; Garnique-Castro y Gutiérrez-Vidrio, 2012; De la Cruz, 2018). Además, hay aún algunos docentes que creen que la inclusión de estudiantes con discapacidad va en detrimento de la educación de aquellos sin discapacidad (Iacoboni y Moirano, 2018) o que sienten incomodidad o inquietud ante las medidas de inclusión (Suriá, 2012).

Igualmente, los docentes refieren dificultades para coordinarse con los maestros de educación especial que acuden a las escuelas para dar apoyo personalizado a estudiantes con discapacidad, lo cual se complica más ante la falta de condiciones institucionales para promover su trabajo colaborativo (Romero y García, 2014). Hay docentes que señalan la necesidad de contar con mayores recursos para garantizar una inclusión efectiva en sus escuelas, una mejor formación inicial para trabajar con estudiantes con alguna discapacidad y más capacitación para atenderles (Arrebola y Villuendas, 2004).

En lo que concierne a los estudiantes, aquellos que no presentan discapacidad también experimentan algunos desafíos cuando estudiantes con discapacidad se incorporan a sus aulas. Suriá (2012) encuentra que, en general, los estudiantes de educación básica tienen actitudes favorables hacia sus compañeros con discapacidad; sin embargo, conforme se aumenta de grado escolar, los docentes perciben que van surgiendo actitudes desfavorables. En educación superior, Salinas (2014) refiere que los factores que más inciden en la buena actitud de estudiantes sin discapacidad hacia la inclusión de compañeros con alguna discapacidad son las emociones generadas por la convivencia con ellos en trabajos en equipo o tutorías, la percepción de que la escuela debe mejorar las condiciones de accesibilidad para estudiantes con discapacidades y la experiencia previa de convivencia con personas con discapacidad.

La resistencia de estudiantes sin discapacidad ante la idea de una educación inclusiva suele ser baja, aunque ya en la práctica surgen algunas barreras que en la investigación educativa se asocian a dificultades para superar los estereotipos sobre discapacidad y desarrollar un entendimiento adecuado de las diferencias y similitudes que existen entre grupos con y sin discapacidades (Cambra, 2005).

Este artículo aporta a la investigación educativa integrando a la discusión a los directores/as escolares, agentes que participan (y en ocasiones lideran) los procesos de toma de decisiones y mejora educativa. Por ello, conocer y entender su participación en la identificación y atención de problemas de educación inclusiva para estudiantes con discapacidad es fundamental.

\section{MARCo anALítico}

En nuestro estudio sobre la situación de la educación inclusiva desde la perspectiva de los directores de EMS en México usamos como marco analítico la Guía para asegurar la inclusión y la equidad en la educación (Unesco, 2018). Este marco es pertinente para esta investigación por dos razones: hace referencia al papel de líderes escolares en la promoción de una educación inclusiva y el apartado de educación inclusiva contenido en el nuevo modelo educativo fue elaborado por la autoridad educativa nacional utilizando este marco de la Organización de las Naciones Unidas para la Educación, la Ciencia y la Cultura (Unesco).

De la Cruz. Educación inclusiva en el nivel medio-superior: análisis desde la perspectiva de directores Sinéctica 54 www.sinectica.iteso.mx 
La definición de educación inclusiva refiere que es necesario hacer frente a las desigualdades de acceso, participación, procesos y resultados del aprendizaje para lograr que los sistemas educativos estén al servicio de todos los estudiantes (Unesco, 2018, p. 12). De acuerdo con la estrategia de la Agenda 2030 para el Desarrollo Sostenible y los Objetivos de Desarrollo Sostenible, esta guía reconoce que hacer de la educación inclusiva una realidad requiere la participación y coordinación de múltiples actores, así como del reconocimiento de las diferencias como un recurso para democratizar y enriquecer aprendizajes (p. 13) y de la apropiación de prácticas y valores alineados con la inclusión para generar y mantener un cambio cultural en las comunidades escolares. El papel de los líderes escolares para este propósito resulta fundamental.

La guía de la Unesco (2018) propone cuatro dimensiones para entender el grado en que los sistemas educativos garantizan la inclusión y la equidad en la educación: conceptos, declaraciones sobre políticas, estructuras y sistemas, y prácticas. Aquí nos enfocamos en una sola dimensión: declaraciones sobre políticas, la cual centra su atención en el papel de los líderes escolares en la promoción y el mantenimiento de prácticas de educación inclusivas. Esta dimensión contiene cuatro subdimensiones que sirven para generar diagnósticos sobre el grado en que los líderes fomentan la educación inclusiva:

-Los documentos importantes de política educativa nacional hacen un fuerte hincapié en la inclusión y la equidad.

-Los altos funcionarios a nivel nacional, de distrito y de escuela lideran en materia de inclusión y equidad en la educación.

- Los líderes en todos los niveles articulan objetivos políticos coherentes para desarrollar prácticas educativas inclusivas y equitativas.

-Los líderes en todos los niveles cuestionan las prácticas educativas no inclusivas, discriminatorias y desiguales.

En resumen, el marco de la Unesco, en particular la dimensión de declaraciones sobre políticas, está alineado con el objetivo de saber en qué medida la educación inclusiva está presente en el trabajo diario de los directores/as de EMS.

\section{MÉTOdos}

\section{Recolección de datos}

Con la finalidad de conocer de primera mano las actividades que realizan los directores/as de la EMS, así como los contextos donde ejercen sus labores y sus necesidades de capacitación, un equipo de investigadoras (en el que participó la autora de este artículo) realizó un estudio exploratorio en 2017 con el apoyo de la Subsecretaría de Educación Media Superior de la Secretaría de Educación Pública (SEP).

Como instrumento de recolección de datos, utilizamos un cuestionario en línea con la herramienta Google Survey. El cuestionario fue enviado por correo electrónico a todos los directores/as de escuelas públicas de EMS en el país y contenía cinco preguntas abiertas y una de opción múltiple, con el propósito de identificar: 
- Ubicación de los planteles, subsistema y entidad de la escuela.

-Características generales de los directores/as: género, participación en el Servicio Profesional Docente, tiempo que había fungido como director/a.

-Las labores y los contextos escolares donde se desempeñan.

Un total de 4,058 directores/as respondieron el cuestionario durante enero de 2017. Con la información, se creó una base de datos y las respuestas se agruparon en cuatro grandes categorías:

-Actividades cotidianas de los directores/as (típicas de lunes y miércoles).

-Acontecimiento o logro que le hubiera generado al director/a orgullo durante los últimos seis meses.

-Acontecimiento o circunstancia que hubiera constituido para el director/a el principal desafío durante los últimos seis meses.

-Situación o suceso que le preocupa al director/a, pero que podría resolverse con capacitación.

\section{Selección y organización de datos}

Si bien los datos de la base sobre competencias directivas no se recabaron mediante preguntas sobre la educación inclusiva, el formato de respuesta libre acerca de situaciones que para ellos son cotidianas, motivo de orgullo o de desafío permite tener una primera aproximación al grado en el que los temas y conceptos de la educación inclusiva son relevantes para quien dirige una escuela de EMS en México. De esta manera, fue posible crear una submuestra de 76 testimonios sobre educación inclusiva para estudiantes con discapacidades.

La submuestra se construyó a través de una búsqueda de testimonios que incluyeran términos asociados con educación a estudiantes con alguna discapacidad. Estos términos emergen de la literatura académica, de documentos de política pública dirigidos a personas con discapacidad y de una revisión preliminar de la base de datos. Es importante señalar que la búsqueda incluyó también palabras que la normativa y la autoridad educativa consideran incorrectas (por ejemplo, "discapacitado" y "discapacitada"); esto se hizo para tratar de incluir en la base todos los testimonios sobre población estudiantil con discapacidad a partir de los términos que componen el discurso de los directores/as. Algunas de las citas en los resultados incluyen estos conceptos incorrectos, pero se presentan sin edición como constancia del discurso que algunos siguen utilizando; los que usamos para construir la submuestra se encuentran en la tabla 1. 


\section{Análisis}

Para el análisis de los testimonios que integraron la submuestra, utilizamos una serie de códigos predeterminados creados con base en los parámetros que establece la Guía para asegurar la inclusión y la equidad en la educación (Unesco, 2018) (ver tabla 2).

Tabla 1. Términos para identificar testimonios sobre el trabajo de directores o directoras con estudiantes con discapacidad

\begin{tabular}{|c|c|c|}
\hline Categoría & Fuente & Términos usados para construir submuestra \\
\hline \multirow{28}{*}{$\begin{array}{l}\text { Discapacidad } \\
\text { física o mental }\end{array}$} & \multirow{28}{*}{$\begin{array}{c}\text {-Moliner } \\
\text {-Unesco } \\
\text {-SEP: nuevo modelo } \\
\text { educativo } \\
\text {-Análisis preliminar } \\
\text { de muestra } \\
\text { - Formato } 911 \text { (SEP) }\end{array}$} & Silla de ruedas \\
\hline & & Discapacidad \\
\hline & & CAED \\
\hline & & Necesidades especiales \\
\hline & & Estudiantes especiales \\
\hline & & Educación especial \\
\hline & & Invidente \\
\hline & & Discapacitada/o \\
\hline & & Ceguera \\
\hline & & Ciego \\
\hline & & Motriz \\
\hline & & Rampa \\
\hline & & Invidente \\
\hline & & Muda/o \\
\hline & & Sorda/o \\
\hline & & Débil visual \\
\hline & & Sordera \\
\hline & & Capacidades diferentes \\
\hline & & Baja visión \\
\hline & & Hipoacusia \\
\hline & & Discapacidad intelectual \\
\hline & & Discapacidad psicosocial \\
\hline & & Sordoceguera \\
\hline & & Trastorno del espectro autista \\
\hline & & Discapacidad múltiple \\
\hline & & Dificultad severa de aprendizaje \\
\hline & & Trastorno por déficit de atención \\
\hline & & Hiperactividad \\
\hline
\end{tabular}

Hubo, además, un código emergente asociado a cuando los directores/as reconocen la presencia de estudiantes con alguna discapacidad en sus planteles, pero sus testimonios no refieren actividades o estrategias específicas para garantizar su inclusión en la comunidad escolar. El nombre de ese código emergente fue "aceptación-reconocimiento pasivo de estudiantes con necesidades diferentes". 
Tabla 2. Códigos con los que se realizó el análisis de testimonios

\begin{tabular}{|c|c|}
\hline Tipo de código & Código \\
\hline & $\begin{array}{c}\text { - Acciones para fomentar desarrollo de prácticas inclusivas } \\
\text { - Sistemas de apoyo que eliminan prácticas no inclusivas } \\
\text { Predeterminados }\end{array}$ \\
$\begin{array}{c}\text { - Sistemas de apoyo que identifican prácticas no inclusivas } \\
\text { - Sistemas de apoyo que desafían prácticas no inclusivas } \\
\text { - Capacitación para fortalecer inclusión }\end{array}$ \\
\hline Emergente & $\begin{array}{c}\text { Aceptación-reconocimiento pasivo de estudiantes con necesi- } \\
\text { dades diferentes }\end{array}$ \\
\hline
\end{tabular}

\section{RESULTADOS}

Educación inclusiva para estudiantes con discapacidad en la EMS

En 76 de las 4,035 escuelas incluidas en la base de datos sobre competencias directivas en la EMS, los directores refieren que parte de su labor involucra la atención a uno o varios estudiantes con alguna discapacidad. Si bien se trata de una proporción menor de escuelas, el propósito de este estudio es brindar una descripción detallada, un primer entendimiento, de las formas que toma el trabajo de los directores/as cuando sus planteles no ponen barreras iniciales de acceso; esto es, cuando se sigue la normativa de inclusión educativa y se matricula a estudiantes con alguna discapacidad en EMS. Las poblaciones con alguna discapacidad que los directores/as identifican y para quienes desarrollan estrategias de inclusión se muestran en la tabla 3.

Tabla 3. Referencia a trabajo con estudiantes con alguna discapacidad

\begin{tabular}{|c|c|c|}
\hline $\begin{array}{c}\text { Término con el que se identifica a } \\
\text { estudiantes con discapacidad }\end{array}$ & Frecuencia total & Porcentaje de frecuencia \\
\hline Silla de ruedas & 6 & $8 \%$ \\
\hline Discapacidad o términos asociados & 19 & $25 \%$ \\
\hline CAED & 15 & $20 \%$ \\
\hline Necesidades especiales & 4 & $5 \%$ \\
\hline Prácticas educativas especiales & 2 & $3 \%$ \\
\hline Discapacidad motriz & 1 & $1 \%$ \\
\hline Ciega/o, ceguera, debilidad visual y \\
términos asociados & 4 & $5 \%$ \\
\hline Sorda/o e hipoacusia & 3 & $4 \%$ \\
\hline Capacidades diferentes & 7 & $9 \%$ \\
\hline Asuntos/problemas/situaciones \\
psicosociales & 12 & $16 \%$ \\
\hline Autismo & 3 & $4 \%$ \\
\hline Totales & 76 & $100 \%$ \\
\hline
\end{tabular}

Fuente: Elaboración propia con datos de la base sobre competencias directivas en EMS.

Respecto a cómo los directores/as enmarcan su trabajo con estudiantes con alguna discapacidad, el análisis muestra que, en el $50 \%$ de las ocasiones, dicho trabajo se presenta como motivo de orgullo, mientras que en el $16 \%$ de las menciones se muestra como un desafío. Asimismo, en el 14\% de los testimonios que hablan de estudiantes con discapacidad, los directores/as hacen referencia a la necesidad de capacitación para brindar un servicio educativo pertinente. En el 
$18 \%$ de los testimonios, el trabajo con algún estudiante con discapacidad es para los directores/as parte de sus actividades rutinarias.

El análisis de los 76 testimonios sobre el trabajo con estudiantes con discapacidades dejó ver que los directores/as diseñan e implementan estrategias de inclusión que, si bien muestran la disposición de derribar barreras, también dejan ver que hay falta de acompañamiento y apoyo por parte de las autoridades educativas estatales y federales.

A continuación, presentamos los hallazgos a la luz de los cuatro componentes de la dimensión "Declaraciones sobre políticas" de la Guía para asegurar la inclusión y equidad en la educación (Unesco, 2018).

\section{Componente i: presencia de principios de inclusión y equidad en documentos} importantes de política educativa nacional

En este rubro, el avance en México es significativo. La Constitución mexicana, la Ley General de Educación y el nuevo modelo educativo establecen que los estudiantes, independientemente de cualquier circunstancia física o social, tienen derecho a una educación de calidad. Sin embargo, las prioridades de política educativa en la práctica no se basan, de modo necesario, en principios de inclusión y equidad para la población con discapacidades. Los testimonios dejan ver que la apertura de los CAED no está acompañada de financiamiento o equipamiento pertinentes. El siguiente testimonio ejemplifica esta situación:

El desafío más complejo hasta ahora es un chico con capacidades diferentes, con problemas para leer. Las escuelas donde lo pudieran apoyar están en la ciudad y, al ser de bajos recursos, no puede acudir. Él estuvo hasta el semestre anterior con nosotros, por ser ésta una escuela incluyente, pero carecemos de herramientas para atender estos casos. [Su caso] se canalizó a la dependencia especializada UNEME en la ciudad y lo están valorando para determinar en qué se le puede apoyar (director en Quintana Roo).

Componente ii: capacitación en responsabilidades para fortalecer la inclusión y eliminar los obstáculos

El análisis de los testimonios deja ver que los directores/as participantes requieren de formación para atender a estudiantes con discapacidades. Si bien hay quienes señalan que tanto ellos como los docentes se preparan para recibir a estudiantes ciegos, sordos y con hipoacusia, hay ocasiones en las que ellos mismos deben cubrir los costos de esas capacitaciones, porque no pueden conseguir fondos de la autoridad educativa para ese propósito.

Recabamos también testimonios que resaltan la necesidad de formación para atender problemas psicosociales, los cuales están vinculados a fenómenos como deserción, violencia escolar, drogadicción, alcoholismo, embarazo, depresión, incapacidad de manejar emociones, hostigamiento y delincuencia.

Si bien el CAED está respaldado por documentos que explican elementos básicos de los tipos de discapacidad que existen (DGB, 2018), una revisión de estos materiales dejó ver que son apenas un primer acercamiento a temas complejos que se 
deben atender por toda la comunidad escolar y, principalmente, por las autoridades educativas. El siguiente testimonio muestra la urgencia de sistemas adecuados de formación: "[Es un desafío] contar con alumnos especiales; ni los maestros ni yo tenemos experiencia en ello y no somos maestros en educación especial. Exhorto ayuda. Los maestros no estamos capacitados, y muchas veces en vez de ayudar podemos perjudicar, ese es mi temor" (directora en Baja California Sur).

Esta necesidad de capacitación pertinente para trabajar con estudiantes con alguna discapacidad es consistente con hallazgos sobre educación inclusiva a poblaciones con discapacidades en nivel primaria (Romero y García, 2014).

\section{Componente iii: fomento de prácticas inclusivas y educativas}

Este componente tuvo la mayor coincidencia con los testimonios de directores. Las formas que toman estas prácticas pueden agruparse en las siguientes categorías:

-Aceptación en el plantel de jóvenes con discapacidad.

-Gestión de recursos (humanos y financieros) para hacer ajustes de infraestructura (baños accesibles, rampas).

-Gestión ante autoridades e instancias privadas para adquirir u obtener como donación equipo o mobiliario para estudiantes (silla de ruedas, auxiliar auditivo, material para lectura en braille).

-Apertura del CAED (planeación, actividades de promoción del centro y arrancar el servicio).

-Adaptación de dinámicas de trabajo e incluso agendas personales para garantizar servicios educativos pertinentes para una estudiante. Un ejemplo de esto es el caso de una directora que brinda servicio de transporte a estudiante en silla de ruedas cuyos padres no pueden llevarlo a la escuela.

-Gestión de becas para estudiantes con discapacidad.

-Integración de estudiantes con discapacidad a actividades culturales (pastorales, altares de muertos) y deportivas.

-Actividades para sensibilizar a estudiantes que son o serán compañeros de estudiantes con alguna discapacidad.

-Gestiones para que estudiantes con discapacidad reciban tratamientos o intervenciones quirúrgicas.

El siguiente testimonio ejemplifica el conjunto de prácticas que algunos directores/as realizan para garantizar una educación inclusiva a estudiantes con discapacidades:

Nuestro plantel es incluyente, aunque no tenemos la infraestructura ni los conocimientos necesarios para atender a alumnas y alumnos sordos, mudos, débiles visuales, ciegos, etc. Es un punto que se trata en las reuniones de academia para acordar cómo tratar casos que reciba el plantel; se analizan y se establecen estrategias para poder ayudar al alumno durante su estancia en la escuela. Junto con los padres de familia, se establece una comunicación y monitoreo constante, se le asignaron compañeros de su salón como monitores, para auxiliarlo en las indicaciones verbales de sus profesores. Incluso uno de ellos aprendió lenguaje para personas mudas. Se considera un caso de éxito, ya que el 
alumno aprobó en ordinario todas sus asignaturas y fue un gran ejemplo para sus compañeros (director en Estado de México).

Categoría iv: sistemas de apoyo que identifican, desafían y eliminan prácticas no inclusivas

El análisis con esta categoría arroja resultados que dejan ver la falta de acompañamiento y apoyo por parte de las autoridades educativas que enfrentan los directores/as. Aunque un testimonio hace referencia a un subsistema que accedió a pagar el salario de un docente que dos veces por semana pudiera trabajar con un estudiante con discapacidad, en la generalidad, los directores/as refieren que el trabajo con estudiantes con discapacidades es difícil porque no se cuenta con las condiciones apropiadas para brindar un servicio educativo de calidad. En este sentido, los testimonios reflejan que hay una doble barrera para alcanzar el entendimiento e inclusión de estudiantes con discapacidades: por un lado, las precarias condiciones sociales y económicas de sus entornos escolares, familiares y personales y, por otro, la falta de programas pertinentes de política educativa.

En general, los hallazgos son consistentes con la literatura sobre educación inclusiva a estudiantes con alguna discapacidad (Flores y García, 2016).

\section{CONCLUSIONES}

Si bien en México se han hecho ajustes legales que reconocen el derecho a una educación inclusiva, independientemente de consideraciones físicas y sociales de los individuos, el discurso aún no se materializa en las escuelas. El análisis de testimonios de directores/as que trabajan con personas con discapacidad revela que el personal escolar reconoce su obligación de brindarles servicios educativos y desarrolla estrategias para garantizarles el derecho a la educación. Sin embargo, los directores/as aclaran también que no cuentan con las habilidades, conocimientos ni recursos para atender las necesidades y asegurar oportunidades educativas para todos.

Estos hallazgos coinciden con otros resultados de investigación sobre educación inclusiva a poblaciones con discapacidad (De la Cruz, 2018). Resolver estas carencias no es cosa sencilla. En términos de formación, los directores/as de EMS en México no son egresados de programas con componentes de pedagogía, gestión o liderazgo, sino que han cursado planes de educación superior diversos. En este sentido, la formación continua resulta fundamental para conocer técnicas, herramientas y teorías que permitan identificar las barreras de la educación que enfrentan sus estudiantes.

Más allá de transferir recursos, que sin duda ayudarían, se trata de ajustes en el sistema educativo para garantizar la atención a las diversas dimensiones de la vida escolar: de organización, infraestructura, formación docente y directiva, prácticas de aula incluyentes, involucramiento de padres de familia, administración de recursos, pero también de relaciones y prácticas entre los miembros de la comunidad de aprendizaje. Se trata de políticas educativas de afirmación positiva para que, desde los aspectos educativos y sociales, aseguren oportunidades de aprendizaje para todos los estudiantes. 
Respecto al diseño de políticas públicas que promuevan la inclusión educativa, parece que el sistema educativo mexicano cuenta con información sobre los estudiantes con discapacidad. Esta información, proveniente del formato 911, de las bases de datos e informes del INEE y de la investigación educativa, parece no ser suficiente -o suficientemente bien administrada- para garantizar que las escuelas reciban recursos (financieros, humanos, de formación) que ayuden a identificar y brindar servicios educativos pertinentes y de calidad.

Para que la educación inclusiva deje de ser discurso e imposición para los directores/as, será necesario atender las condiciones estructurales desfavorables. Como se señala en el marco propuesto por la Unesco, la inclusión educativa requiere acciones en todos los niveles y de cada uno de los actores que componen e interactúan con las comunidades escolares. Cargar semejante responsabilidad a un solo actor, en este caso los directores/as de nivel medio superior en México, es sinónimo de desentendimiento por parte de las autoridades educativas.

\section{REFERENCIAS BIBLIOGRÁFICAS}

Arrebola, I. A. y Villuendas Giménez, M. D. (2004). Las actitudes del profesorado hacia el alumnado con necesidades educativs especiales. Convergencia, pp. 183-215.

Cambra, C. (2005). Percepción de la sordera y la integración por parte del alumnado sin necesidades educativas especiales. Educar, pp. 155-168.

Constitución Política de los Estados Unidos Mexicanos (15 de agosto de 2018). Artículo $3^{\circ}$. Recuperado del Instituto de Investigaciones Jurídicas de la UNAM: https://www.juridicas.unam.mx/legislacion/ordenamiento/constitucionpolitica-de-los-estados-unidos-mexicanos

De la Cruz Flores, G. (2018). Inclusión y discapacidad en educación media superior: experiencias docentes. En Debates en Evaluación y Curriculum. Congreso Internacional de Educación (pp. 1-10). Tlaxcala: Universidad Autónoma de Tlaxcala-Posgrado en Educación de la Facultad de Ciencias de la Educación.

DGB (11 de julio de 2018). Curso de inducción a CAED. Recuperado de Dirección General de Bachillerato: https://sites.google.com/a/dgb.email/caedcapacitacion/

Flores Barrera, V. J. y García Cedillo, I. (2016). Apoyos que reciben estudiantes de secundaria con discapacidad en escuelas regulares: ¿corresponden a lo que dicen las leyes? Educación, pp. 1-20.

Garnique-Castro, F. y Gutiérrez-Vidrio, S. (2012). Educación básica e inclusión: un estudio de representaciones sociales. Revista Internacional de Investigación en Educación, pp. 577-593.

Iacoboni, G. y Moirano, A. M. (2018). Reflexiones acerca de la inclusión de alumnos con discapacidad en la clase de lengua. Relen, Revista Estudios de Lenguas (Universidad Nacional de Salta), pp. 103-116.

INEE (2019). Panorama educativo de México. Indicadores del Sistema Educativo Nacional 2018. Educación básica y media superior. Ciudad de México.

INEE (febrero de 2018). Condiciones básicas para la enseñanza y el aprendizajeven los planteles de educación media superior en México. Resultados generales. Recuperado de Instituto Nacional para la Evaluación de la Educación: https://publicaciones.inee.edu.mx/buscadorPub/P1/D/247/P1D247.pdf 
INEE (2 de febrero de 2018). La educación obligatoria en México: Informe 2018. Recuperado de https://www.inee.edu.mx/portalweb/informe2018/04_informe/capitulo_010303.html

INEE (2017). Directrices para mejorar la permanencia escolar en la educación media superior. México.

Ley General de Educación (19 de enero de 2018). Secretaría de Educación Pública. Recuperado de https://www.sep.gob.mx/work/models/sep1/ Resource/558c2c24-0b12-4676-ad90-8ab78086b184/ley_general_educacion.pdf

Moliner García, O. (2013). Educación inclusiva. Castelló de la Plana: Publicacions de la Universitat Jaume I.

Romero Contreras, S. y García Cedillo, I. (2014). Educación especial en México. Desafíos de la educación inclusiva. Revista Latinoamericana de Educación Inclusiva, pp. 77-91.

Salinas Alarcón, M. (2014). Actitudes de estudiantes sin discapacidad hacia la inclusión de estudiantes con discapacidad en la educación superior. Barcelona, España: Universidad Autónoma de Barcelona.

Sanhueza Enríquez, S., Granada Azcárraga, M. y Bravo Cóppola, L. (2012). Actitudes del profesorado de Chile y Costa Rica hacia la inclusión educativa. Cuadernos de Pesquisa, pp. 884-899.

SEP (11 de julio de 2018). Centros de Atención para Estudiantes con Discapacidad. Recuperado de Secretaría de Educación Pública: https://www.dgb.sep. gob.mx/servicios-educativos/caed/index.php

SEP (2017). Modelo educativo para la educación obligatoria: educar para la libertad y la creatividad. México.

Suriá Martínez, R. (2012). Discapacidad e integración educativa: ¿qué opina el profesorado sobre la inclusión de estudiantes con discapacidad en sus clases? Revista Española de Educación y Psicopedagogía, pp. 96-109.

Unesco (2018). Guía para asegurar la inclusión y la equidad en la educación. París: Organización de las Naciones Unidas para la Educación, la Ciencia y la Cultura.

UNICEF (2016). Niños y niñas fuera de la escuela. México.

Varas Giler, R. C. (2018). Percepción de los profesores sobre la inclusión. Integrando en el aula de clases a estudiantes universitarios con necesidades especiales. En R. R. Vanessa Karina Barreiro Fonseca. Estrategias educativas y sociales para la inclusión de la discapacidad (pp. 50-62). Guayaquil: Centro de Investigación y Desarrollo Ecuador. 